\title{
Study on Influence of Cognitive Load for Software Developer's Performance using BPNN Algorithm
}

\author{
K.Banu \\ Research Scholar \\ Mother Teresa Women's University \\ Kodaikanal, Tamilnadu
}

\author{
N.Rama, PhD \\ Research Supervisor \\ Presidency College \\ Chennai -5
}

\begin{abstract}
The development process involved developers contribution based his/her cognitive thinking in the real time process. The developer's performance is dynamic as per their cognitive load. The cognitive load is un-deterministic as well hidden and integrated in the developer's process. This paper attempt to identify software developer cognitive measure which influences the development process using neural network back propagation model .It describes the conceptual view on conventional construction of neural network for cognitive measure observation of software development processes. A neural network model designed to present the structure of developer's performance such as Regularity, Task Completion, Accuracy, Team Involvement and Reporting are used to generate the Performance and Cognitive Load of the output layer. To obtain the Performance and the Cognitive Load from the given input, the Cognitive work load such as physical ability, mental ability, temporal ability, effort, frustration and performance are assigned to a hidden layer. The observation and the results are described and discussed as part of the paper.
\end{abstract}

\section{Keywords}

Cognitive load, Performance, Neural network, Back Propagation, Influence factor

\section{INTRODUCTION}

The software development process is integrated and improved with its architecture, functional analysis and the development process resources. The cognitive models are used for the computations to empower the process and its applications[1].The development process are highly involved the contribution of developers based on their thinking and acting intelligence[2]. The developer performance reflected the development of software and its quality. The developer's intellectual capacity influenced the cognitive measures and its loads $[3,4]$.The cognitive process of the developers are created major impact on the quality of the software therefore this paper aimed to design a model to determine the influencing cognitive factors to the software developers performance. Using statistical measures, how much the cognitive load influences the performance of the software developer's is not predictable in the developer's process [.5]. The cognitive models are determined simulating the neural computations process [6]. They related to cogitation and human performance related learning and its impact are evaluated to improve the human behavior [ 7,8]. The model construction for cognitive process introduced by Luce for psychology [9] and based on the evaluations of Massaro [10].The model was used for multiple functionality of human by Meyer in 1997[11]. In continuing the process Rigier[12] and Ritter [13] attempted to design mathematical models for computational process. The theoretical aspects and its computational are addressed by R.sun $[14,15,16]$. It leads the basic computational and biological properties of individual neurons and networks of neurons, which give rise to basic processing mechanisms like spreading activation, inhibition, and multiple constraint satisfaction. It examine a range of cognitive and emotional phenomena within this framework, including attention, memory, language, higher-level cognition, motivation, emotion, and personality which creates an impact on their performance. Therefore, this paper adopts the neural network model to evaluate the performance of the software developers and overcome the inadequacy of the statistical basic measures.

\section{NEURAL NETWORK FOR COMPUTING DEVELOPER COGNITION}

The neural network based learning [17, 18] is a process adopted the layer based approach. This model comes under the prediction system. It is used to determine the developers performance based on their cognitive load. It is used to forecast some future values of the input data. It provides the option for the identification of developer's skill in different multidisciplinary domains according to their cognitive load and performance. With the help of this back propagation neural network model the developer's performance as well as the quality of the product can be improved.

\subsection{Back propagation algorithm}

It is systematic method of training multi-layer artificial neural networks [19]. A Back propagation network consists of three layers of units, an input layer, at least one hidden layer, and an output layer. Units are connected in a feed-forward fashion with input units fully connected to hidden layer and hidden units fully connected to units in the output layer. When a back propagation is cycled an input pattern is forwarded to the output units through the intervening input-to-hidden and hidden-to-output weights. The application of the generalized delta rule thus involves two phases: During the first phase the input is presented and propagated forward through the network to compute the output values for each output unit. This output is compared with its desired value do, resulting in an error signal for each output unit. The second phase involves a backward pass through the network during which the error signal is passed to each unit in the network and appropriate weight changes are calculated.

As per the adopted back propagation neural network model, the observed data is mapped and the model is constructed. The adopted algorithm is converted as a step by step executable concept and presented below 
Step 1:

Normalize the input and output with respect to their maximum values. For each training pair, assume that in normalized form there are

$$
\begin{array}{r}
\ell \text { inputs given by }\{\mathrm{I}\} \text { I and } \\
\ell \times 1 . \\
\mathrm{n} \text { outputs given by }\{\mathrm{O}\} \mathrm{O} \\
\mathrm{n} \times 1
\end{array}
$$

Step 2:

Assume that the number of neurons in the hidden layers lie between $1<\mathrm{m}<10$.

Step 3:

Let [V] represents the weights of synapses connecting input neuron and hidden neuron. Let [W] represents the weights of synapses connecting hidden neuron and output neuron. Initialize the weights to small random values usually from -1 to +1 ;

$$
\begin{aligned}
{[\mathrm{V}]^{0} } & =[\text { random weights }] \\
{[\mathrm{W}]^{0} } & =[\text { random weights }]
\end{aligned}
$$

$[\Delta \mathrm{W}]^{0}=[0]$

$$
\Delta[\mathrm{V}]^{0}=
$$

For general problems $\lambda$ can be assumed as 1 and threshold value as 0 .

\section{Step 4:}

For training we need to present one set of inputs and outputs. Present the pattern as inputs to the input layer $\{\mathrm{I}\} \mathrm{I}$ then by using linear activation function, the output of the input layer may be evaluated as.

$$
\{0\} \mathrm{I}=\{\mathrm{I}\} \mathrm{I}
$$

$\ell \times 1$.

$\ell \times 1$

Step 5: Compute the inputs to the hidden layers by multiplying corresponding weights of synapses as

$$
\{\mathrm{I}\} \mathrm{H}=[\mathrm{V}] \mathrm{T}\{\mathrm{O}\} \mathrm{I}
$$

$$
\mathrm{m} \times \ell . \ell \times 1 \text {. }
$$

$\mathrm{m} \times 1$.

Step 6: Let the hidden layer units, evaluate the output using the sigmoidal function as

$$
\{\mathrm{O}\} \mathrm{H}=\left\{\frac{1}{1+\mathrm{e}-(\mathrm{IHI})}\right\}
$$

$\mathrm{m} \times 1$

Step 7: Compute the inputs to the output layers by multiplying corresponding weights of synapses as

$$
\begin{aligned}
& \{\mathrm{I}\} \mathrm{O}=[\mathrm{W}] \mathrm{T}\{\mathrm{O}\} \mathrm{H} \\
& \mathrm{nx} 1 \mathrm{nxm} \operatorname{mx} 1
\end{aligned}
$$

Step 8: Let the output layer units, evaluate the output using sigmoidal function as

$$
\{0\}_{0}=\left\{\frac{1}{1+\mathrm{e}-(\mathrm{IOj})}\right\}
$$

Note: This output is the network output

Step 9: Calculate the error using the difference between the network output and the desired output as for the jth training set as

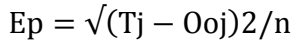

Step 10: Find a term $\{d\}$ as

$$
\{\mathrm{d}\}=(\mathrm{Tk}-\text { Ook }) \text { Ook }(1-\text { Ook })
$$

$\mathrm{n} \times 1$

Step 11: Find [ Y ] matrix as

$$
\begin{aligned}
& {[\mathrm{Y}]=\{\mathrm{O}\} \mathrm{H}\{\mathrm{d}\}} \\
& \operatorname{mxn} \operatorname{mx} 11 \times n
\end{aligned}
$$

Step 12: Find

$$
\begin{aligned}
& {[\Delta \mathrm{W}] \mathrm{t}+1=\alpha[\Delta \mathrm{W}] \mathrm{t}+\eta[\mathrm{Y}]} \\
& \operatorname{mxn} \quad \operatorname{mxn} \quad \operatorname{mxn}
\end{aligned}
$$

Step 13: Find

$$
\begin{aligned}
& \{\mathrm{e}\}=[\mathrm{W}]\{\mathrm{d}\} \\
& \mathrm{mx} 1 \quad \mathrm{mxn} n \times 1 \\
& \left(\mathrm{O}_{\mathrm{Hi}}\right)\left(1-\mathrm{O}_{\mathrm{Hi}}\right) \\
& \{\mathrm{d} *\}=\mathrm{ei}
\end{aligned}
$$$$
\mathrm{mx} 1 \quad \mathrm{~m} \times 1
$$

Find $[\mathrm{X}]$ matrix as

$$
\begin{array}{ll}
\{\mathrm{d} *\} & {[\mathrm{X}]=\{\mathrm{O}\} \mathrm{I}\left\{\mathrm{d}^{*}\right\}=\{\mathrm{I}\} \mathrm{I}} \\
1 \mathrm{xm} & 1 \times \mathrm{xm} \quad \ell \times 11 \times \mathrm{x} \quad \ell \times 1
\end{array}
$$

Step 14: Find

]

$$
[\Delta \mathrm{V}] \mathrm{t}+1=\alpha[\Delta \mathrm{V}] \mathrm{t}+\eta[\mathrm{X}
$$$$
1 \times \mathrm{m} \quad 1 \times \mathrm{m}
$$

$1 \times \mathrm{m}$

Step 15: Find

$$
\begin{gathered}
{[\mathrm{V}] \mathrm{t}+1=[\mathrm{V}] \mathrm{t}+[\Delta \mathrm{V}] \mathrm{t}+1} \\
{[\mathrm{~W}] \mathrm{t}+1=[\mathrm{W}] \mathrm{t}+[\Delta \mathrm{W}] \mathrm{t}+1}
\end{gathered}
$$

Step 16: Find error rate as

$$
\text { Error rate }=\mathrm{Ep} / \mathrm{nset}
$$

Step 17: Repeat steps 4 to 16 until the convergence in the error rate is less than the tolerance value.

The Back propagation algorithm is converted for determining the influence factors of the software developers

\subsection{Model for back propagation algorithm}

A neural network is a powerful data-modeling tool that is able to capture and represent complex input/output relationships $[19,20]$. It is based on supervised learning since every input pattern is used to train the network. The input layer designed with the selection of domain, project and the phases involved, with the basic inputs needed to evaluate an employee called Regularity, Task Completion, Accuracy, Team Involvement and Reporting. The Cognitive work load such as physical ability, mental ability, temporal ability, effort, frustration and performance and their cross-functional specification process are assigned to the hidden layer. Learning process is based on the comparison between the network computed output and the expected correct output. The error generated is used to change 
the network parameters that result improved performance. As per the network structure developers industrial observations and their cognitive work load are used to generate the Performance and Cognitive Load of the output layer. Since the developer's skills are multidimensional it improves the performance of the developer's as well as the quality of the product.
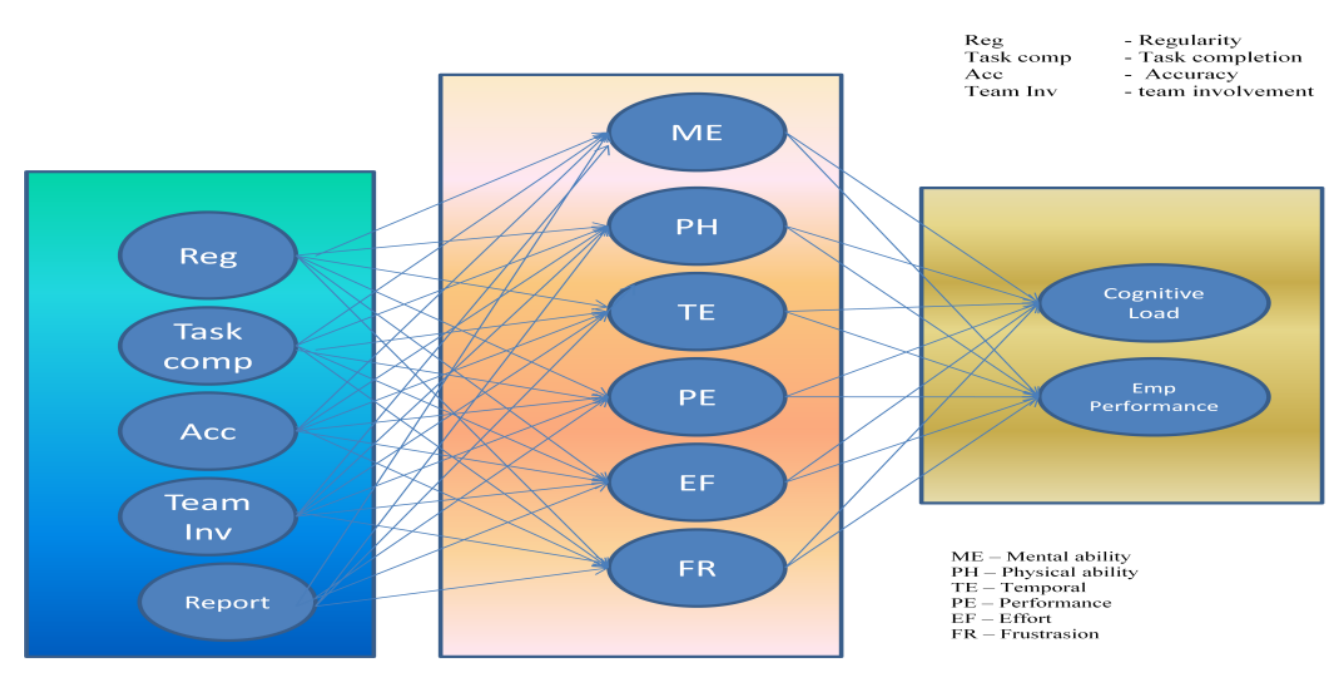

Fig 1: Neural Network model for Developers Performance determination

\section{IMPLEMENTATION AND RESULT ANALYSIS}

There are five input attribute weights are presented in line to the six hidden neurons. The weight values from $1^{\text {st }}$ row to $5^{\text {th }}$ row represents regularity, task completion, accuracy, team involvement and reporting of the input layer which interacted with Mental Demand, Physical Demand, Temporal
Demand, Performance, Effort and Frustration weight values The value Vo denotes the weight value of the input layer to the hidden layer in Table1. The hidden layer to the output layer values are states as Wo. There are two output columns which represents the cognitive load and the performance. The six internal attributes produce the weighted value of output Wo presented on the Table2.

Table1: Initial Vo weight values

\begin{tabular}{|r|r|r|r|r|r|r|}
\hline $\begin{array}{r}\text { Cognitive load } \\
\text { /External Factors }\end{array}$ & $\begin{array}{r}\text { Mental } \\
\text { Demand }\end{array}$ & $\begin{array}{r}\text { Physical } \\
\text { demand }\end{array}$ & $\begin{array}{r}\text { Temporal } \\
\text { Demand }\end{array}$ & Performance & Effort & Frustration \\
\hline Regularity & 1.629447 & 0.195081 & 0.315226 & 0.283773 & 1.311481 & 1.51548 \\
\hline Task Completion & 1.811584 & 0.556996 & 1.941186 & 0.843523 & 0.071423 & 1.486265 \\
\hline Accuracy & 0.253974 & 1.093763 & 1.914334 & 1.831471 & 1.698259 & 0.784454 \\
\hline Team Involvement & 1.826752 & 1.915014 & 0.970751 & 1.584415 & 1.867986 & 1.310956 \\
\hline Reporting & 1.264718 & 1.929777 & 1.600561 & 1.918985 & 1.35747 & 0.342373 \\
\hline
\end{tabular}

Table 2: Initial computed Wo values

\begin{tabular}{|l|c|c|}
\hline Cognitive load/ Output & Cognitive Load & Performance \\
\hline Mental Demand & 4.640872 & 4.629654 \\
\hline Physical Demand & 3.966658 & 4.251925 \\
\hline Temporal Demand & 4.211748 & 4.885047 \\
\hline Performance & 3.980997 & 3.969272 \\
\hline Effort & 4.031957 & 4.37357 \\
\hline Frustration & 4.758283 & 4.316384 \\
\hline
\end{tabular}


As pre the observation of table 2, first column maximum value 4.75 denotes frustration influenced on the Cognitive Load. At the same time the second column maximum value Temporal Demand 4.88 influenced the Performance. But the values of the influences are changed during the iteration and the optimized values are obtained. The maximum values of internal cognitive loads factors are consider as an influencing factor for the external factors. The obtained values and its influencing impacts are discussed further.

\section{DISCUSSION ON COMPUTED COGNITIVE FACTOR VALUES}

Employee Cognitive (Internal) load is depending on external factors weights values. As per the observation on above presented obtained NN percentage weights the following are derived

- Regulatory influenced by frustration .The influencing percentage on frustration by regularity is $27.36 \%$
- Temporal demand weights are forced based on task completion and accuracy. The Temporal demand forced $28.79 \%$ on Task Completion and $28.39 \%$ on accuracy

- Tem involvement and reporting depends on physical demand. The team involvement dependency is $33.65 \%$ and reporting is 33.91 according to physical demand

- Regularity minimum value of $3.43 \%$ applied for physical demand

- Task completion minimum percentage 1.13 least influenced for effort cognitive load

- The accuracy minimum percentage 3.75 least affected on mental demand

- Team involvement minimal value 14.4 made the minimum force on temporal demand

- Frustration percentage value 6.29 is affected at the minimal according to the report

Table 3: External variables and Cognitive weight values (Input-to-Hidden)

\begin{tabular}{|l|c|c|c|c|c|c|}
\hline Cognitive load /External Factors & $\begin{array}{c}\text { Mental } \\
\text { Demand }\end{array}$ & $\begin{array}{c}\text { Physical } \\
\text { demand }\end{array}$ & $\begin{array}{c}\text { Temporal } \\
\text { Demand }\end{array}$ & Performance & Effort & Frustration \\
\hline Regularity & 24.01 & 3.43 & 4.68 & 4.39 & 20.80 & 27.86 \\
\hline Task Completion & 26.69 & 9.79 & 28.79 & 13.05 & 1.13 & 27.32 \\
\hline Accuracy & 3.74 & 19.22 & 28.39 & 28.34 & 26.93 & 14.42 \\
\hline Team Involvement & 26.92 & 33.65 & 14.40 & 24.52 & 29.62 & 24.10 \\
\hline Reporting & 18.64 & 33.91 & 23.74 & 29.70 & 21.52 & 6.29 \\
\hline
\end{tabular}

Table 4: Cognitive weight values and output variables (Hidden-to-Output)

\begin{tabular}{|c|c|c|}
\hline Cognitive load/ Output & Cognitive Load & Performance \\
\hline Mental Demand & 18.13512 & 17.51941 \\
\hline Physical Demand & 15.5005 & 16.09002 \\
\hline Temporal Demand & 16.45824 & 18.48587 \\
\hline Performance & 15.55653 & 15.02041 \\
\hline Effort & 15.75567 & 16.55035 \\
\hline Frustration & 18.59393 & 16.33394 \\
\hline
\end{tabular}

The cognitive load and performance are based on the developer's internal factors. The maximum value of cognitive load $18.59 \%$ is observed for the frustration and the performance influence 18.48 according to the temporal demand weight values. The cognitive load is not affected much because of the physical demand minimum value 15.50 $\%$. The overall performance is also not majorly disturbed based on the cognitive performance minimum value of 15.02 $\%$.

\section{CONCLUSION}

A neural network model with 5:6:2 designed to present the structure of developer's performance such as Regularity, Task
Completion, Accuracy, Team Involvement and Reporting are used to generate the Performance and Cognitive Load of the output layer. The Performance and the Cognitive Load from the given input, the Cognitive work load such as physical ability, mental ability, temporal ability, effort, frustration and performance are assigned to a hidden layer. The observation and the results show that the cognitive load as an internal factor which influences the external parameters. The internal component interaction weigh values are determined between Input layers, output layer via hidden layer. Thus The Back Propagation neural network model helps to identify software developer cognitive measure which influences the development process. 


\section{REFERENCES}

[1] M. Dawson, (2002). Computer modelling of cognition: Levels of analysis. In:Nadel, L. (ed.), Encyclopedia of Cognitive Science. pp. 635-638. Macmillan,London, UK.

[2] Brain Drain: Evaluating the Impact of Increased Cognitive Load During Self-Paced Running Performance, J McCarron, TL Hodgson, MF Smith, Published in British Journal of sports Medicine, 2013.

[3] Analysis of Developers Cognitive Complexity Association for software development K.Banu , Research Scholar, Mother Teresa Women's University , Kodaikanal, Tamilnadu \& Dr.N.Rama, Research Supervisor, Presidency College, Chennai -5

[4] Comparative Study on Multidimensional Developers Performance with Cognitive Load K.Banu , Research Scholar, Mother Teresa Women's University , Kodaikanal, Tamilnadu \& Dr.N.Rama, Research Supervisor, Presidency College, Chennai -5

[5] Software Developers Performance relationship with Cognitive Load Using Statistical Measures K.Banu , Research Scholar, Mother Teresa Women's University, Kodaikanal, Tamilnadu \& Dr.N.Rama, Research Supervisor, Presidency College, Chennai -5

[6] P. Dayan, (2003). Levels of analysis in neural modeling. In: L. Nadel (ed.),Encyclopedia of Cognitive Science. Macmillan, London.

[7] S. Grossberg, (1982). Studies of Mind and Brain: Neural Principles of Learning, Perception, Development, Cognition, and Motor Control. Norwell, MA: Kluwer Academic Publishers.

[8] D. Hintzman, (1990). Human learning and memory: Connections and dissociations. In: Annual Review of Psychology, pp.109-139. Annual Reviews Inc, Palo Alto, CA.

[9] R.D.Luce ,(1995) .Four tensions concerning mathematical modeling in psychology. Annual Review of Psychology, 46, 1-26. Annual Reviews Inc, PaloAlto, CA.
[10] D. Massaro, (1988). Some criticisms of connectionist models of human performance. Journal of Memory and Language, 27, 213-234.

[11] D. Meyer and D. Kieras, (1997). A computational theory of executive cognitive processes and human multipletask performance: Part 1, basic mechanisms.Psychological Review. 104 (1), 3-65.

[12] T. Regier, (2003). Constraining computational models of cognition. In: L.Nadel (ed.), Encyclopedia of Cognitive Science. Macmillan, London. pp.611-15.

[13] F. E. Ritter, Shadbolt, N., Elliman, D., Young, R., Gobet, F., and Baxter,G., (2003). Techniques for Modeling Human Performance in Synthetic Environments: A Supplementary Review. Human Systems Information Analysis Center, Wright-Patterson Air Force Base, Dayton, $\mathrm{OH}$.

[14] R. Sun, (2005). Theoretical status of computational cognitive modeling. Technical report, Cognitive Science Department, Rensselaer Polytechnic Institute,Troy, New York.

[15] R. Sun (ed.), (2006). Cognition and Multi-Agent Interaction: From Cognitive Modeling to Social Simulation. Cambridge University Press, New York.

[16] R. Sun, (2007). The importance of cognitive architectures: An analysis based on CLARION. Journal of Experimental and Theoretical Artificial Intelligence, in press.

[17] J. Hertz, A. Krogh, R.G. Palmer, Introduction to the Theory of Neural Computation, Addison-Wesley, Reading, MA, 1991

[18] D.P. Bertsekas, J.N. Tsitsiklis, Neuro-Dynamic Programming, Athena Scientific, Belmont, MA, 1996.

[19] Neural Networks on the NetBeans Platform , Zoran Severac published by Oracle, Feb 2011.

[20] Mohsen Hayati, Yazdan Shirvany Artificial Neural Network Approach for Short Term Load Forecasting, International Journal of Electrical, Computer and Systems Engineering Volume 1, Number 2007. 